\title{
ON ALGEBRAS ASSOCIATED TO PARTIALLY ORDERED SETS
}

\author{
MORTEN BRUN and TIM RÖMER
}

\begin{abstract}
We continue the work [2] on sheaves of rings on finite posets. We present examples where the ring of global sections coincide with toric faces rings, quotients of a polynomial ring by a monomial ideal and algebras with straightening laws. We prove a rank-selection theorem which generalizes the well-known rank-selection theorem of Stanley-Reisner rings. Finally, we determine an explicit presentation of certain global rings of sections.
\end{abstract}

\section{Introduction}

In the present paper we continue the work [2] on sheaves of commutative rings on finite partially ordered sets (posets for short).

A finite poset $P$ can be considered as a topological space with the Alexandrov topology [1], that is, the topology where the open sets are the subsets $U$ of $P$ such that $y \in U$ and $x \leq y$ implies $x \in U$.

A presheaf $\mathscr{T}$ on $P$ consists of an abelian group $\mathscr{T}(U)$ for every open subset $U$ of $P$ and restriction maps $\mathscr{T}_{V U}: \mathscr{T}(U) \rightarrow \mathscr{T}(V)$ for $V \subseteq U$ open subsets of $P$. The restriction maps are subject to the conditions that $\mathscr{T}_{U U}$ is the identity on $\mathscr{T}(U)$ and that $\mathscr{T}_{W V} \circ \mathscr{T}_{V U}=\mathscr{T}_{W U}$ for $W \subseteq V \subseteq U$ open subsets of $P$. The stalk $T_{x}$ of $\mathscr{T}$ at $x \in P$ is the set $\bigsqcup_{x \in U} \mathscr{T}(\bar{U}) / \sim$ of equivalence classes represented by elements $t \in \mathscr{T}(U)$ for $U$ an open subset of $P$ containing $x$ under the equivalence relation generated by requiring $t \sim \mathscr{T}_{V U}(t)$ whenever $V \subseteq U$ are open subsets of $P$ containing $x$. The presheaf $\mathscr{T}$ is a sheaf if the sequence

$$
0 \rightarrow \mathscr{T}\left(U_{1} \cup U_{2}\right) \rightarrow \mathscr{T}\left(U_{1}\right) \times \mathscr{T}\left(U_{2}\right) \rightarrow \mathscr{T}\left(U_{1} \cap U_{2}\right)
$$

is exact for every pair $\left(U_{1}, U_{2}\right)$ of open subsets of $P$. Here the map $\mathscr{T}\left(U_{1} \cup\right.$ $\left.U_{2}\right) \rightarrow \mathscr{T}\left(U_{1}\right) \times \mathscr{T}\left(U_{2}\right)$ is $\left(\mathscr{T}_{U_{1} U_{1} \cup U_{2}}, \mathscr{T}_{U_{2} U_{1} \cup U_{2}}\right)$, and the map $\mathscr{T}\left(U_{1}\right) \times$ $\mathscr{T}\left(U_{2}\right) \rightarrow \mathscr{T}\left(U_{1} \cap U_{2}\right)$ is given by $\mathscr{T}_{U_{1} \cap U_{2} U_{1}}$ on the first factor and by $-\mathscr{T}_{U_{2} \cap U_{2} U_{1}}$ on the second factor.

Received January 2, 2006; in revised form March 7, 2007. 
A sheaf $\mathscr{T}$ on $P$ is uniquely determined by its stalks $\left(T_{x}\right)_{x \in P}$ and the homomorphisms $T_{x y}: T_{y} \rightarrow T_{x}$ for $x \leq y$ in $P$ induced by the restriction maps of $\mathscr{T}$. More precisely, the stalks of $\mathscr{T}$ form a $Z P$-algebra $T$, that is, a system $\left(T_{x}\right)_{x \in P}$ of abelian groups and homomorphisms $T_{x y}: T_{y} \rightarrow T_{x}$ for $x \leq y$ in $P$ with the property that $T_{x x}$ is the identity on $T_{x}$ and that $T_{x y} \circ T_{y z}=T_{x z}$ for every $x \leq y \leq z$ in $P$. Conversely, there is a sheaf $\mathscr{T}$, with $\mathscr{T}(U)$ equal to the (inverse) limit $\lim _{x \in U} T_{x}$, associated to every $\mathrm{Z} P$-algebra $T$. Here $\lim _{x \in U} T_{x}$ is the subgroup of the product $\prod_{x \in U} T_{x}$ consisting of families $\left(f_{x}\right)_{x \in U}$ with $T_{x y}\left(f_{y}\right)=f_{x}$ for all $x \leq y$ in $U$. This defines a one to one correspondence between sheaves on $P$ and $Z P$-algebras, and thus the concepts of sheaves and of $Z P$-algebras are equivalent.

Let $R$ be a $Z^{m}$-graded commutative Noetherian ring. By abuse of terminology we say that $\mathscr{T}$ is a sheaf of $Z^{m}$-graded $R$-algebras if $\mathscr{T}$ is a sheaf on $P$ and the restriction maps $\mathscr{T}_{U V}$ are homomorphisms of $Z^{m}$-graded $R$-algebras. Similarly we say that $T$ is an RP-algebra if the homomorphisms $T_{x y}$ are homomorphisms of $Z^{m}$-graded $R$-algebras. The above discussion also shows that the concept of sheaves of $Z^{m}$-graded $R$-algebras is equivalent to the concept of $R P$-algebras. The $R P$-algebra $T$ is called flasque if the associated sheaf $\mathscr{T}$ is flasque, that is, if the restriction map $\mathscr{T}_{V U}: \mathscr{T}(U) \rightarrow \mathscr{T}(V)$ is surjective for every inclusion $V \subseteq U$ of open subsets of $P$.

In this paper we study the ring $H^{0}(P, \mathscr{T}):=\mathscr{T}(P)=\lim _{P} T$ and call it the ring of global sections of $T$. Under this name it was for example studied by Yuzvinsky [22], [23] and Caijun [6]. We are particularly interested in $R P$ algebras that appear in the literature on algebraic combinatoric and commutative algebra, including face rings for fans and simplicial complexes, monomial ideals and algebras with straightening laws. Applying results from [2] on local cohomology of $H^{0}(P, \mathscr{T})$ we obtain a rank selection theorem in the tradition of Duval [8], Hibi [10], Munkres [13] and Stanley [15]. In order to state it we need a little terminology. Firstly, in [10] Hibi calls a subset $X$ of $P$ excellent if for every element $z$ of $P$ there exists at most one $x \in X$ with $x \leq z$. Secondly, the rank of $P$ is the supremum of the numbers $|C|-1$ for $C$ a chain in $P$, and the rank of $x \in P$ is the rank of the poset $\left(0_{\widehat{P}}, x\right]=\{z \in P: z \leq x\}$. Thirdly, if there exists exactly one $Z^{m}$-graded ideal $\mathfrak{m}$ in $R$ such that $R / \mathfrak{m}$ is a field, then we call $R$ a $Z^{m}$-graded local ring. Note that $R / \mathrm{m}$ is concentrated in degree zero, and thus $\mathfrak{m}$ contains every element of $R$ of non-zero degree. In particular, if $K=R_{0}$ is a field, then $\mathrm{m}=\bigoplus_{a \in Z^{m} \backslash\{0\}} R_{a}$. Finally, we denote by $H_{\mathfrak{m}}^{i}(N)$ the local cohomology modules of a finitely generated $\mathrm{Z}^{m}$-graded $R$-module $N$ with respect to $\mathfrak{m}$ (see [5] for details). Since $\mathfrak{m}$ is a maximal ideal the definition of graded local cohomology modules and usual local cohomology modules coincide. With the notation recollected in Definition 4.1, the following generalizes Hibi's result [10, Theorem 1.7]. 
THeOREM 1.1. Let $(R, \mathfrak{m})$ be a $\mathrm{Z}^{m}$-graded local ring with $K=R_{0}$ a field and let $X$ be an excellent subset of a finite poset $P$. Let $T$ be a flasque $Z^{m}$-graded $R P$-algebra with $\operatorname{dim}_{\lim _{P} T} T=\operatorname{rank}(P)$, and such that the homomorphism $R \rightarrow \lim _{P} T$ is surjective. Assume that:

(i) $T_{y}$ is Cohen-Macaulay of dimension $\operatorname{rank}(y)$ for every $y \in P$.

(ii) For every $x \in X$ we have that $\operatorname{rank}(\operatorname{star}(x))=\operatorname{rank}(P)$ and we have that $\operatorname{rank}\left(\operatorname{star}(x) \cap P_{X}\right)=\operatorname{rank}(P)-1$.

(iii) For every $x \in X$ and $y \in \operatorname{star}(x)$ we have that $\widetilde{H}^{i}\left(\left(y, 1_{\operatorname{star}(x)}\right) ; K\right)=0$ for $i \neq \operatorname{rank}(P)-\operatorname{rank}(y)-1$.

(iv) For every $x \in X$ and $y \in \operatorname{star}(x) \cap P_{X}$ we have $\widetilde{H}^{i}\left(\left(y, 1_{\operatorname{star} \widehat{x}) \cap P_{X}}\right) ; K\right)=0$ for $i \neq \operatorname{rank}(P)-\operatorname{rank}(y)-2$.

Then the natural homomorphism

$$
H_{\mathfrak{m}}^{i}\left(\lim _{P} T\right) \rightarrow H_{\mathfrak{m}}^{i}\left(\lim _{P_{X}} T\right)
$$

is surjective for $i=\operatorname{rank}(P)$, it is injective for $i=\operatorname{rank}(P)-1$, and otherwise it is an isomorphism.

Here the cohomology groups $\widetilde{H}^{i}(P ; K)$ are reduced singular cohomology groups of the underlying topological space of the poset $P$, or equivalently the cohomology groups of the associated order complex.

As an application of Theorem 1.1 we consider the following situation. We define the $i$ th skeleton of a finite poset $P$ to be the sub-poset $P^{(i)}=\{y \in P$ : $\operatorname{rank}(y) \leq i\}$. If for example $P=P(\Sigma)$ is the face poset of a rational pointed fan $\Sigma$ in $\mathrm{R}^{m}$ and $T=T(\Sigma)$ is the $K P$-algebra as constructed in Example 2.1, then we obtain that

$$
\text { depth } \lim _{P} T=\max \left\{i \in \mathrm{N}: i \leq \operatorname{rank}(P) \text { and } \lim _{P^{(i)}} T \text { is Cohen-Macaulay }\right\} .
$$

If $P$ is the face poset of a simplicial complex $\Delta$ and $T$ is the Stanley-Reisner $K P$-algebra associated to $\Delta$, then this is a well-known result, because $\lim T \cong$ $K[\Delta]$ is the Stanley-Reisner ring associated to $\Delta$ and $\lim _{P^{(i)}} T \cong K\left[\Delta^{(i)}\right]$ is the Stanley-Reisner ring associated to the $i$ th skeleton $\Delta^{(i)}$ of $\Delta$ in this situation.

We end the paper by discussing presentations of $R P$-algebras. In Theorem 5.6 we give a criterion, namely that $T$ is sectioned and has a presentation $\Pi$, ensuring that the $\operatorname{ring} \lim _{P} T$ of global sections of an $R P$-algebra $T$ is of the form $K[F] / I$ for a finite set $F$, where the ideal $I$ is a sum $I=I_{T}+I_{\Delta_{\Pi}}$ of the Stanley-Reisner ideal of a simplicial complex $\Delta_{\Pi}$ related to the order complex of $P$ and the sum $I_{T}$ of defining ideals for the stalks $T_{x}$ of $T$. A presentation 
of $T$ roughly consists of a subset $F_{x}$ of $F$ and a surjective homomorphism $K\left[F_{x}\right] \rightarrow T_{x}$ for every $x$ in $P$. Using the main result of [2] again we obtain the following description of the depth and the dimension of certain $K P$-algebras. ( $K$ is a field.)

COROLlaRY 1.2. Let $(\Pi, p)$ be a presentation of a sectioned flasque KPalgebra $T$ consisting of $Z^{m}$-graded Cohen-Macaulay rings such that $d_{x}<d_{y}$ and $f_{x}<f_{y}$ for $x<y$ in $P$, where $d_{x}=\operatorname{dim}\left(T_{x}\right)$ and $f_{x}=\left|F_{x}\right|$. We have that

$$
\begin{aligned}
\operatorname{depth} \lim _{P} T & =\min \left\{i \in \mathrm{N}: H_{\mathfrak{m}}^{i+\left(f_{x}-d_{x}\right)}\left(K\left[\Delta_{\Pi}\right]\right) \neq 0 \text { for some } x \in P\right\}, \\
\operatorname{dim} \lim _{P} T & =\max \left\{i \in \mathrm{N}: H_{\mathfrak{m}}^{i+\left(f_{x}-d_{x}\right)}\left(K\left[\Delta_{\Pi}\right]\right) \neq 0 \text { for some } x \in P\right\} .
\end{aligned}
$$

In particular, if there exists an $n \in \mathbf{Z}$ such that $\operatorname{dim}\left(K\left[F_{x}\right]\right)=\operatorname{dim}\left(T_{x}\right)+n$ for every $x \in P$ and such that $\operatorname{dim}\left(K\left[\Delta_{\Pi}\right]\right)=\operatorname{dim}\left(\lim _{P} T\right)+n$, then $\lim _{P} T$ is Cohen-Macaulay if and only if $\Delta_{\Pi}$ is Cohen-Macaulay.

The paper is organized as follows: In Section 2 we present classes of $K P$ algebras associated to toric face rings (as introduced in [3]), monomial ideals and algebras with straightening laws (ASL's for short). In Section 3 we give a criterion on a $K P$-algebra with stalks given by ASL's to have an ASL as ring of global sections. As an application we generalize a construction of Stanley [17] who defined the face ring of a simplicial poset. In Section 4 we prove Theorem 1.1 and study some of its consequences. Finally, in Section 5 we study presentations of $K P$-algebras.

\section{Examples of $R P$-algebras}

In this section we present $R P$-algebras appearing naturally in algebraic combinatoric and commutative algebra. Of course every $K$-algebra $S$ equals $\lim _{P} T$ for the poset $P=\{x\}$ consisting of one element and the $K P$-algebra $T$ with $T_{x}=S$. However this gives no new information about $S$. Our goal is to choose a finite poset $P$ and a suitable $K P$-algebra $T$ such that the stalks $T_{x}$ are as nice as possible (e.g. Cohen-Macaulay rings) and to characterize ring properties of $\lim _{P} T$ in terms of combinatorial properties of $P$ and ring properties of the rings $T_{x}$ for $x \in P$. Our first example goes back to a construction of Stanley [16].

EXAMPLE 2.1 (Toric face rings). We consider a rational fan $\Sigma$ in $\mathrm{R}^{m}$, that is, $\Sigma$ is a finite collection of rational cones in $\mathrm{R}^{m}$ such that for $C^{\prime} \subseteq C$ with $C \in \Sigma$ we have that $C^{\prime}$ is a face of $C$ if and only if $C^{\prime} \in \Sigma$, and if $C, C^{\prime} \in \Sigma$, then $C \cap C^{\prime}$ is a common face of $C$ and $C^{\prime}$. For each cone $C \in \Sigma$ we choose an affine monoid $M_{C} \subseteq \mathrm{Z}^{m}$ such that: 
(i) cone $\left(M_{C}\right)=C$ for $C \in \Sigma$;

(ii) If $C, C^{\prime} \in \Sigma, C^{\prime} \subseteq C$, then $M_{C^{\prime}}=M_{C} \cap C^{\prime}$.

Observe that we do not require that $M_{C}$ is normal. Let $P(\Sigma)=(\Sigma, \subseteq)$ be the partially ordered set of faces of $\Sigma$ ordered by inclusion and let $K$ be a field. For $C \in P(\Sigma)$ we let $T_{C}$ denote the affine monoid ring $K\left[M_{C}\right]$. For $C^{\prime} \subseteq C$ in $P(\Sigma)$ the homomorphisms $T_{C^{\prime} C}: T_{C} \rightarrow T_{C^{\prime}}$ are induced by the natural face projections $K\left[C \cap \mathrm{Z}^{m}\right] \rightarrow K\left[C^{\prime} \cap \mathrm{Z}^{m}\right]$. This is a $Z^{m}$-graded $K P$ algebra, and with $R=\lim T$ it is an $R P$-algebra of $Z^{m}$-graded $R$-algebras. We write $T=T(\Sigma)$ if $M_{C}=C \cap \mathrm{Z}^{m}$, for every $C \in \Sigma$. In this case the ring $\lim T(\Sigma)$ is called the toric face ring of $\Sigma$, and it was studied by Stanley [16], Bruns-Gubeladze [4] and Brun-Römer [3].

Stanley-Reisner rings of finite simplicial complexes are covered by the above example: To a simplicial complex $\Delta$ we associate a fan $\Sigma$ with the same face poset as $\Delta$, and we can choose $M_{C}$ isomorphic to $\mathrm{N}^{\operatorname{dim}(C)}$ for every $C \in \Sigma$. In this situation we write $P(\Delta)=P(\Sigma)$ and call $T(\Delta)=T(\Sigma)$ the StanleyReisner $K P$-algebra. The ring $\lim T(\Delta)$ is isomorphic to the Stanley-Reisner ring $K[\Delta]$. (See [2, Example 5.2].)

Our next example shows that rings defined by monomial ideals give rise to $K P$-algebras.

Example 2.2 (Monomial ideals). Let $S=K\left[x_{1}, \ldots, x_{n}\right]$ be the polynomial ring over a field $K$ with the usual $\mathrm{Z}^{n}$-grading. Recall that irreducible monomial ideals in $S$ are of the form $\mathrm{m}^{b}=\left(x_{1}^{b_{1}}, \ldots, x_{n}^{b_{n}}\right)$ for $0 \neq b \in \mathbf{N}^{n}$. A monomial ideal $I$ has a unique irredundant irreducible decomposition of the form

$$
I=\mathfrak{m}^{b^{1}} \cap \cdots \cap \mathfrak{m}^{b^{t}} \quad \text { for } \quad b^{1}, \ldots, b^{t} \in \mathbf{N}^{n} .
$$

(See [11, Section 5.2] for details.) For each subset $C$ of $[t]=\{1, \ldots, t\}$ there exists a unique maximal subset $\bar{C} \subseteq[t]$ with the property that $\sum_{i \in C} \mathfrak{m}^{b^{i}}=$ $\sum_{i \in \bar{C}} \mathfrak{m}^{b^{i}}$. Let $P(I)$ denote the poset of the subsets $\bar{C}$ of $[t]$ for $\emptyset \neq C \subseteq[t]$ ordered by reverse inclusion. For $C \in P(I)$ let $T(I)_{C}=S / \sum_{i \in C} \mathfrak{m}^{b^{i}}$ and for $C, D \in P(I)$ with $C \subseteq D$ we define $T(I)_{D C}: T_{C} \rightarrow T_{D}$ to be the natural projection map. Now:

(i) $T(I)$ is a $Z^{n}$-graded $S P(I)$-algebra.

(ii) By using Example 3.3 of [2] we see that $T(I)$ is flasque with $\lim T(I) \cong$ $S / I$.

(iii) $T(I)_{C}$ is a complete intersection and thus Cohen-Macaulay for $C \in$ $P(I)$. 
The result [2, Theorem 4.1] has the following corollary. (See [20, Theorem 1] for a more general description of $H_{\mathfrak{m}}^{i}(S / I)$.)

Corollary 2.3. Let $I=\mathfrak{m}^{b^{1}} \cap \cdots \cap \mathfrak{m}^{b^{t}} \subset K\left[x_{1}, \ldots, x_{n}\right]$ be an irredundant irreducible decomposition of a monomial ideal and let $d_{C}=\operatorname{dim} T(I)_{C}$. If $d_{C}>d_{D}$ for $C, D \in P(I)$ with $D \subset C$ then $H_{\mathfrak{m}}^{i}\left(K\left[x_{1}, \ldots, x_{n}\right] / I\right)$ is isomorphic to

$$
\bigoplus_{F \in P(I)} \widetilde{H}^{i-d_{F}-1}\left(\left(F, 1_{\widehat{P}(I)}\right) ; K\right) \otimes_{K} H_{\mathfrak{m}}^{d_{F}}\left(K\left[x_{1}, \ldots, x_{n}\right] / \sum_{i \in F} m^{b^{i}}\right)
$$

as a $\mathrm{Z}^{n}$-graded $K$-vector space.

For the next example we recall the notion of an algebra with straightening law (ASL for short). We call a function $m: P \rightarrow \mathrm{N}$ (i.e. $m \in \mathrm{N}^{P}$ ) a monomial on $P$, and consider it as an element of the polynomial ring $K[P]$ on the elements of $P$. The support of $m$ is the set $\operatorname{supp}(m)=\{x \in P: m(x) \neq 0\}$. The monomial is called a standard monomial if $\operatorname{supp}(m)$ is a chain in $P$. Let $R$ be an algebra over a field $K$ with an injection $\phi: P \rightarrow R$. We associate to each monomial $m$ the element $\phi(m)=\prod_{x \in P} \phi(x)^{m(x)} \in R$ and call $\phi(m)$ a monomial in $R$. Following [7] we call $R$ an algebra with straightening law (ASL for short) on $P^{\text {op }}$ over $K$ if

ASL1 The set of standard monomials is a $K$-basis of $R$, and

ASL2 if $x$ and $y$ are incomparable in $P$ and if

$$
\phi(x) \phi(y)=\sum_{m} r_{m, x y} \phi(m)
$$

is the unique representation of $\phi(x) \phi(y)$ as a linear combination of standard monomials guaranteed by ASL1, then $r_{m, x y} \neq 0$ implies that the maximal element $x_{m}$ of $\operatorname{supp}(m)$ satisfies $x<x_{m}$ and $y<x_{m}$.

Note that for technical reasons we work with $P^{\text {op }}$ instead of $P$. By ASL1 every monomial $\phi(n)$ in $R$ has a unique representation of the form $\phi(n)=$ $\sum_{m \in \mathbb{N}^{p}} r_{m, n} \phi(m)$, where $r_{m, n} \neq 0$ implies that $m$ is a standard monomial. We write

$$
r(n)=\sum_{m \in \mathbb{N}^{P}} r_{m, n} m
$$

for the element in the polynomial ring $K[P]$ associated to this representation of $\phi(m)$.

Example 2.4 (ASL). Let $R$ be an ASL on $P^{\text {op }}$. Then $R=K[P] / I_{P}$ where the ideal $I_{P} \subseteq K[P]$ is generated by the straightening relations $n-r(n)$. For 
$Q^{\prime} \subseteq Q$ an inclusion of subsets of $P$ we consider the projection map

$$
p_{Q^{\prime} Q}: K[Q] \rightarrow K\left[Q^{\prime}\right], x \mapsto \begin{cases}x & \text { if } x \in Q^{\prime}, \\ 0 & \text { if } x \notin Q^{\prime}\end{cases}
$$

We let $I_{Q}=p_{Q P}\left(I_{P}\right) \subseteq K[Q]$, we define $T_{Q}=K[Q] / I_{Q}$ and we let $T_{Q^{\prime} Q}: T_{Q} \rightarrow T_{Q^{\prime}}$ denote the map induced by $p_{Q^{\prime} Q}$. For $Q$ open in $P$ the ideal $I_{Q}$ is generated by the elements $n-p_{Q P}(r(n))$ for $n \in \mathrm{N}^{Q}$. By [7, Proposition 1.2(b)] (with $I=P \backslash Q$ ) the $K$-algebra $T_{Q}$ is an ASL on $Q^{\mathrm{op}}$.

Now we let $T_{x}=T_{\left[0_{\widehat{P}}, x\right]}$ for $x \in P$ and $T_{x y}=T_{\left(0_{\widehat{P}}, y\right]\left(0_{\hat{P}}, x\right]}$ for $x, y \in P$ with $x \leq y$. It follows that $T$ is an $R P$-algebra. Using that $T_{Q}$ is an ASL on $Q^{\text {op }}$ if $Q \subseteq P$ is open, we see by working with standard monomials that $\lim _{P} T \cong T_{P}$.

\section{3. $K P$-algebras with an ASL-structure}

We saw in Example 2.4 that an ASL $R$ can be seen as the ring of global sections of a suitable $K P$-algebra. Sometimes it is possible to reverse this construction in the sense that giving locally ASL's one can construct a $K P$-algebra such that the global ring of sections is again an ASL with prescribed presentation.

Recall that $K[P]=K[x: x \in P]$ denotes the polynomial algebra on the elements $x$ of $P$. At first we present a criterion which ensures that a given $K P$-algebra has an ASL-structure.

Theorem 3.1. Let $T$ be a KP-algebra on a finite poset $P$ such that $T_{x}$ is an ASL on $\left(0_{\widehat{P}}, x\right]^{\text {op }}$ for every $x \in P$. Let $\phi_{x}: K\left[\left(0_{\widehat{P}}, x\right]\right] \rightarrow T_{x}$ be the natural projection induced by the ASL-structure on $T_{x}$ for $x \in P$. If the diagram

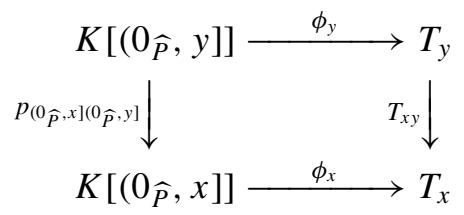

commutes for every $x, y \in P$ with $x \leq y$, then $\lim _{P} T$ is an ASL on $P^{\mathrm{op}}$.

In particular, if $\mathscr{S} \subseteq \mathrm{N}^{P}$ denotes the set of standard monomials in $K[P]$ then $\lim _{P} T \cong K[P] / I_{P}$, where the ideal $I_{P}$ is generated by relations of the form

$$
n-\sum_{m \in \mathscr{S}} r_{m, n} m \quad \text { for } n \in \mathbf{N}^{P} .
$$

Proof. Let $\phi: K[P] \rightarrow \lim _{P} T$ denote the homomorphism induced by the natural projections $K[P] \rightarrow K\left[\left(0_{\widehat{P}}, x\right]\right]$ for $x \in P$. 
We claim that $\phi$ is surjective and moreover $\lim _{P} T$ is generated as a $K$ vector space by the elements $\phi(m)$ with $m \in \mathscr{S}$.

Let $s=\left(s_{x}\right)_{x \in P} \in \lim _{P} T$. Let $x \in P$ and note that $s_{x} \in T_{x}=\lim _{\left.0_{\widehat{P}}, x\right]} T$. Since $T_{x}$ is an ASL on $\left(0_{\widehat{P}}, x\right]^{\text {op }}$ there exist unique scalars $\lambda_{m, s}^{x} \in K$ such that

$$
s_{x}=\sum_{m \in \mathbb{N}^{\left[0 \widehat{P}_{p}, x\right]} \cap \mathscr{S}} \lambda_{m, s}^{x} \phi_{x}(m) .
$$

For every chain $m$ in $P$ we set $x_{m}=\max (\operatorname{supp}(m))$. Note that by the assumptions we have that $\lambda_{m, r}^{x}=\lambda_{m, r}^{y}$ for all $x, y \in P$ such that $y \geq x$ and $x_{m} \leq x, y$. We define $r_{m, s}=\lambda_{m, s}^{x_{m}, r}$. Let

$$
f_{s}=\sum_{m \in \mathscr{S}} r_{m, s} m \in K[P]
$$

Then by the choice of the coefficients $r_{m, s}$

$$
\phi\left(f_{s}\right)=s \in \lim _{P} T .
$$

This shows the first claim. Observe that if $x, y \in P$ are incomparable, then $x_{m}>x, y$ for all $m$ such that $r_{m, \phi(x y)} \neq 0$. Thus ASL2 follows ones we have proved that the standard monomials are $K$-linearly independent in $\lim _{P} T$. Assume that

$$
0=\sum_{m \in \mathscr{S}} a_{m} \phi(m) \in \lim _{P} T .
$$

Choose $n \in \mathscr{S}$ and consider the projection $\pi(T)_{x_{n}}: \lim _{P} T \rightarrow T_{x_{n}}$. Hence

$$
0=\sum_{m \in \mathscr{S}} \pi(T)_{x_{n}}\left(a_{m} \phi(m)\right)=\sum_{m \in \mathbb{N}^{\left[\left(\hat{P}_{\hat{P}}, x_{n}\right]\right.} \cap \mathscr{S}} a_{m} \phi_{x_{n}}(m) \in T_{x_{n}} .
$$

Since $T_{x_{n}}$ is an ASL $\left(0_{\widehat{P}}, x_{n}\right)^{\text {op }}$, we get that $a_{n}=0$. This shows that the standard monomials are indeed $K$-linearly independent in $\lim _{P} T$ and thus we have proved that ASL1 holds. As noted above also ASL2 holds and this shows that $\lim _{P} T$ is an ASL on $P^{o p}$.

EXAMPLE 3.2 (Locally distributive lattices). We refer to [19] for the notion of distributive lattice. A finite poset $P$ is called a locally distributive lattice, if $P$ has a terminal element $0_{P}$ and for all elements $z \in P$ the interval $\left[0_{P}, z\right]$ is a distributive lattice. For example the simplicial posets as considered in [8], [12] and [18], i.e. $P$ has $0_{P}$ and for all $z \in P$ the interval $\left[0_{P}, z\right]$ is a boolean algebra, are locally distributive lattices. Let $z \in P$. Since $\left[0_{P}, z\right]$ is a distributive lattice it follows from a result of Hibi (see [9]) that

$$
T_{z}=K\left[\left[0_{P}, z\right]\right] /(x y-(x \wedge y)(x \vee y))
$$


is a graded ASL on $P^{\mathrm{op}}$ with straightening relation $r_{z}(x, y)=(x \wedge y)(x \vee y)$ (which is an integral domain). Here $\wedge$ and $\vee$ are the meet and join in the distributive lattice $\left[0_{P}, z\right]$. Note that $x \wedge y$ is the meet of $x$ and $y$ in $P$ and $x \vee y$ is a minimal upper bound of $x, y$ in $P$ depending on $z$.

Let $R=K[P]$. The restriction homomorphisms $T_{z^{\prime} z}: T_{z} \rightarrow T_{z^{\prime}}$ for $z, z^{\prime} \in$ $P$ with $z^{\prime} \leq z$, define a Z-graded $K P$-algebra. We call $\lim T$ the generalized Hibi ring associated to $P$. By Theorem $3.1 \lim _{P} T$ is an ASL on $P^{\text {op }}$ and it is easy to see that for incomparable $x, y \in P$ the straightening relations are

$$
r(x, y)=(x \wedge y) \sum_{z} z
$$

where $z$ ranges over all minimal upper bounds for $x, y$. The sum is defined to be zero if there are no such elements.

Note that if $R$ is a graded ASL, i.e. $R$ is a graded $K$-algebra and all elements of $P$ are homogeneous and have positive degree, then it is known that the straightening relation (1) gives a presentation of $R$ (see Proposition 1.1 in [7]). This is not true in the general case (see [14] and [21] for counterexamples).

Together with this observation we proved in Example 3.2 in fact the following corollary:

Corollary 3.3. Let $P$ be a locally distributive lattice and let $T$ be the $K P$-algebra constructed in 3.2. Then $\lim _{P} T$ is a Z-graded ASL on $P^{\mathrm{op}}$ and for $\lim _{P} T=K[P] / I_{T}$ we have that the ideal $I_{T}$ is generated by

$$
x y-(x \wedge y) \sum_{z} z
$$

where $z$ ranges over all minimal upper bounds for $x, y$. The sum is defined to be zero if there are no such elements.

Hence we obtain exactly the poset ring $\tilde{A}_{P}$ constructed by Stanley in [17] for simplicial posets with the same ASL structure. One immediately gets the following result:

Corollary 3.4. Let $P$ and $T$ be as in Corollary 3.3. If $P$ is a CohenMacaulay poset, i.e. $\Delta(P)$ is Cohen-Macaulay simplicial complex, then $\lim _{P} T$ is a Cohen-Macaulay ring.

Proof. Since $\lim _{P} T$ is Z-graded and the elements of $P$ have positive degree, it follows from [7, Cor. 7.2] that $\lim _{P} T$ is a Cohen-Macaulay ring. 


\section{Quotients of rings of global sections}

Let $P$ be a finite poset and $T$ be a flasque $R P$-algebra. Given an open subset $Q$ of $P$, the restriction of $T$ to $Q$ induces a surjective homomorphism

$$
\lim _{P} T \rightarrow \lim _{Q} T .
$$

Thus $\lim _{Q} T$ is a quotient of $\lim _{P} T$. We are interested in the relationship between ring properties of these two rings. In general there is no close connection, but in special situations there is more hope.

The goal of this section is to generalize so-called rank-selection theorems in the tradition of Duval [8], Hibi [10], Munkres [13] and Stanley [15] to our situation. We follow the ideas of Hibi [10] and at first we define:

DEFINITION 4.1.

(i) Let $X$ be a subset of $P$. We denote by $P_{X}$ the sub-poset $P \backslash\left(\bigcup_{x \in X}\left[x, 1_{\widehat{P}}\right)\right)$.

(ii) For $x \in P$ the star of $x$ in $P$ is the sub-poset $\operatorname{star}_{P}(x)$ consisting of the elements $y \in P$ such that there exists $z \in P$ with $x, y \leq z$.

(iii) A non-empty subset $X \subseteq P$ is called excellent if for every element $z$ of $P$ there exists at most one $x \in X$ with $x \leq z$.

Given an element $x \in P$ we let $I_{x}$ denote the kernel of the homomorphism

$$
\lim _{P} T \rightarrow \lim _{P \backslash[x, \widehat{1})} T .
$$

Lemma 4.2. Let $R$ be a $Z^{m}$-graded ring, $X$ be an excellent subset of a finite poset $P$ and $T$ be a flasque $Z^{m}$-graded RP-algebra. Then there exists a short exact sequence

$$
0 \rightarrow \bigoplus_{x \in X} I_{x} \rightarrow \lim _{P} T \rightarrow \lim _{P_{X}} T \rightarrow 0
$$

of $\mathbf{Z}^{m}$-graded $R$-modules.

Proof. The homomorphism

$$
\bigoplus_{x \in X} I_{x} \rightarrow \lim _{P} T
$$

is the sum of the inclusions $I_{x} \rightarrow \lim _{P} T$. This homomorphism is injective because $X$ is excellent. It follows from the definition of $P_{X}$ that the sequence in question is a zero-sequence. It is right-exact, since $T$ is flasque. We leave it as an exercise to the reader to check middle-exactness.

We can now apply the main result in [2]. 
LEMMA 4.3. Let $(R, \mathfrak{m})$ be a $\mathrm{Z}^{m}$-graded local ring with $K=R_{0}$ a field and let $P$ be a finite poset. Let $T$ be a flasque $Z^{m}$-graded RP-algebra such that the homomorphism $R \rightarrow \lim _{P} T$ is surjective. Assume that:

(i) $T_{y}$ is Cohen-Macaulay of dimension $\operatorname{rank}(y)$ for every $y \in P$.

(ii) For every $y \in P$ we have that

$$
\widetilde{H}^{i}\left(\left(y, 1_{\widehat{P}}\right) ; K\right)=0 \quad \text { for } i \neq \operatorname{rank}(P)-\operatorname{rank}(y)-1 .
$$

Then $\lim _{P} T$ is Cohen-Macaulay with $\operatorname{dim} \lim _{P} T=\operatorname{rank}(P)$. In particular, if $P$ is a Cohen-Macaulay poset then the assumption (ii) is satisfied.

Proof. By [2, Theorem 4.1] we have that

$$
H_{\mathfrak{m}}^{i}\left(\lim _{P} T\right) \cong \bigoplus_{y \in P} \tilde{H}^{i-\operatorname{rank}(y)-1}\left(\left(y, 1_{\widehat{P}}\right) ; K\right) \otimes_{K} H_{\mathfrak{m}}^{\operatorname{rank}(y)}\left(T_{y}\right)
$$

as $Z^{m}$-graded $K$-vector spaces. By assumption (ii) we have that

$$
\begin{aligned}
\widetilde{H}^{i-\operatorname{rank}(y)-1}\left(\left(y, 1_{\widehat{P}}\right) ; K\right)= & 0 \\
\text { for } \quad & i-\operatorname{rank}(y)-1 \neq \operatorname{rank}(P)-\operatorname{rank}(y)-1 .
\end{aligned}
$$

The latter condition is equivalent to $i \neq \operatorname{rank}(P)$. Hence

$$
H_{\mathfrak{m}}^{i}\left(\lim _{P} T\right)=0 \quad \text { for } \quad i \neq \operatorname{rank}(P)
$$

and thus $\lim _{P} T$ is Cohen-Macaulay of dimension $\operatorname{rank}(P)$.

We are ready to prove Theorem 1.1.

Proof of Theorem 1.1. For every $x \in X$ the sets $\operatorname{star}(x)$ and $\operatorname{star}(x) \cap P_{X}$ are open subsets of $P$ and thus the ranks of elements in these poset coincide with the ones in $P$. Let $J_{x}$ be the kernel of the surjective homomorphism $\lim _{\text {star }(x)} T \rightarrow \lim _{\operatorname{star}(x) \cap P_{X}} T$. Since $\operatorname{star}(x) \cap P_{X}=\operatorname{star}(x) \backslash\left[x, 1_{\widehat{P}}\right)$ we get a commutative diagram of $R$-modules of the form

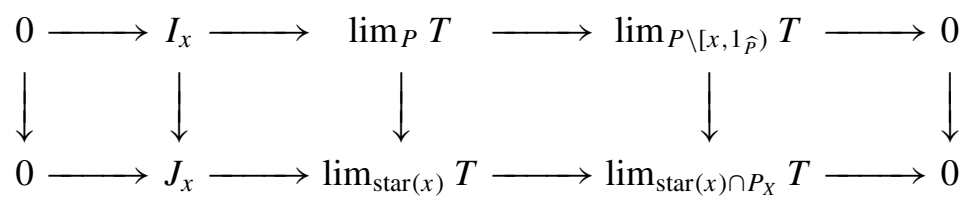

Note that $\lim _{P} T \rightarrow \lim _{\operatorname{star}(x)} T$ and $\lim _{P \backslash\left[x, 1_{\widehat{P}}\right)} T \rightarrow \lim _{\mathrm{star}(x) \cap P_{X}} T$ are surjective and that $I_{x} \rightarrow J_{x}$ is an isomorphism.

By Lemma 4.3 the rings $\lim _{\operatorname{star}(x)} T$ and $\lim _{\operatorname{star}(x) \cap P_{X}} T$ are Cohen-Macaulay of dimension $\operatorname{rank}(\operatorname{star}(x))=\operatorname{rank}(P)$ and $\operatorname{rank}\left(\operatorname{star}(x) \cap P_{X}\right)=\operatorname{rank}(P)-$ 
1 respectively. The long exact local cohomology sequence induced by the second row above shows that $J_{x}$ and thus $I_{x}$ are Cohen-Macaulay of dimension $\operatorname{rank}(P)$.

Next we consider the short exact sequence

$$
0 \rightarrow \bigoplus_{x \in X} I_{x} \rightarrow \lim _{P} T \rightarrow \lim _{P_{X}} T \rightarrow 0
$$

and read the statement of the theorem off from the associated long exact local cohomology sequence.

If $X$ is an excellent subset of a finite poset $P$, then $P_{X}$ is called hereditary if the assumptions (ii)-(iv) of Theorem 1.1 are satisfied. In view of the above result and a theorem of Hochster implying that the normal monoid ring $K\left[D \cap Z^{d}\right]$ is Cohen-Macaulay of dimension $d$ for every $d$-dimensional rational pointed cone $D$ in $\mathrm{R}^{d}$, we obtain the following

Corollary 4.4. Let $P(\Sigma)$ be the face poset of a rational pointed $d$ dimensional fan $\Sigma$ in $\mathrm{R}^{m}$, let $R=\lim _{P} T$ and let $T(\Sigma)$ be the associated $K P(\Sigma)$-algebra of Example 2.1. If $X$ is a hereditary subset of $P(\Sigma)$ then the homomorphism

$$
H_{\mathfrak{m}}^{i}\left(\lim _{P(\Sigma)} T\right) \rightarrow H_{\mathfrak{m}}^{i}\left(\lim _{P(\Sigma)_{X}} T\right)
$$

is surjective for $i=d$, it is injective for $i=d-1$, and otherwise it is an isomorphism.

A sub-poset $Q$ of $P$ is called $n$-hereditary (or hereditary for short) if there exists a sequence $X_{1}, X_{2}, \ldots, X_{n}$ of subsets of $P$ and a sequence $Q=P_{1} \subseteq$ $P_{2} \subseteq \cdots \subseteq P_{n+1}=P$ of sub-posets of $P$ such that $X_{i}$ is an excellent subset of $P_{i+1}$ and $P_{i}=\left(P_{i+1}\right)_{X_{i}}$ hereditary in $P_{i+1}$ for $i=1, \ldots, n$.

Corollary 4.5. Let $Q$ be an $n$-hereditary sub-poset of a finite poset $P$. Let $(R, \mathfrak{m})$ be a $\mathrm{Z}^{m}$-graded local ring with $R_{0}=K$ a field and let $T$ be a flasque $\mathrm{Z}^{m}$-graded RP-algebra such that the homomorphism $R \rightarrow \lim _{P} T$ is surjective and such that $T_{y}$ is Cohen-Macaulay of dimension $\operatorname{rank}(y)$ for every $y \in P$. Then

$$
H_{\mathfrak{m}}^{i}\left(\lim _{P} T\right) \cong H_{\mathfrak{m}}^{i}\left(\lim _{Q} T\right) \quad \text { for } \quad i=0, \ldots, \operatorname{rank}(P)-1-n
$$

as $\mathrm{Z}^{m}$-graded $\lim _{P} T$-modules. In particular, if $\lim _{P} T$ is Cohen-Macaulay and $\operatorname{rank}(Q)=\operatorname{rank}(P)-n$, then $\lim _{Q} T$ is Cohen-Macaulay.

The $i$ th skeleton of a poset $P$ is the sub-poset

$$
P^{(i)}=\{y \in P: \operatorname{rank}(y) \leq i\} .
$$


EXAmple 4.6. Let $P=P(\Sigma)$ be the face poset of a rational pointed fan $\Sigma$ in $\mathrm{R}^{m}$ and let $T=T(\Sigma)$ be the $K P(\Sigma)$-algebra of Example 2.1. We claim that $P^{(i)}$ is a hereditary sub-poset of $P^{(i+1)}$ with respect to the excellent subset

$$
X^{(i)}=\left\{C \in P^{(i+1)}: \operatorname{rank}(C)=i+1\right\} .
$$

For $C \in X^{(i)}$ we have that $\operatorname{star}(C) \cap P^{(i+1)}$ contains its supremum, and thus it is contractible. For every $C \in P$ the poset $\operatorname{star}(C) \backslash\{C\}$ is the face poset of the boundary of a cone and thus Cohen-Macaulay. This shows the claim.

We obtain immediately from Corollary 4.5 that

$$
\text { depth } \lim _{P} T=\max \left\{i \in \mathrm{N}: i \leq \operatorname{rank}(P) \text { and } \lim _{P^{(i)}} T \text { is Cohen-Macaulay }\right\} .
$$

If $P$ is the face poset of a simplicial complex $\Delta$ and $T$ is the Stanley-Reisner $K P$-algebra associated to $\Delta$, then this is a well-known result.

\section{Presentations of sectioned $K P$-algebras}

Recall that for a set $F$ we denote by $K[F]$ the polynomial ring on the variables $a \in F$ and that the support of a monomial $m \in K[F]$ is the $\operatorname{set} \operatorname{supp}(m)=$ $\{a \in F: m(a)>0\}$. Given an inclusion $F_{x} \subseteq F$ of finite sets we denote by $\pi_{x}: K[F] \rightarrow K\left[F_{x}\right]$ the natural projection with $\pi_{x}(a)=a$ if $a \in F_{x}$ and $\pi_{x}(a)=0$ otherwise. There is a $K P$-algebra $T^{\Pi}$ associated to every system $\Pi=\left(F_{x}\right)_{x \in P}$ of subsets of a finite set $F$ with $F_{x} \cap F_{y}=\cup_{z \leq x, y} F_{z}$ for every $x, y \in P$ defined by $\left(T^{\Pi}\right)_{x}=K\left[F_{x}\right]$ and $\left(T^{\Pi}\right)_{x y}(f)=\pi_{x}(f)$ for $x \leq y$ in $P$ and $f \in K\left[F_{y}\right] \subseteq K[F]$. The projections $\pi_{x}: K[F] \rightarrow$ $K\left[F_{x}\right]$ induce a surjective homomorphism $\pi: K[F] \rightarrow \lim _{P} T^{\Pi}$ with kernel $\operatorname{Ker}(\pi)=\bigcap_{x \in P} \operatorname{Ker}\left(\pi_{x}\right)$ equal to the ideal generated by the set of monomials $m$ in $K[F]$ such that $\operatorname{supp}(m)$ is not contained in any of the sets $F_{x}$. Thus the ring $\lim _{P} T^{\Pi}$ is isomorphic to the Stanley-Reisner ring of the simplicial complex $\Delta_{\Pi}$ consisting of the subsets $G$ of $F$ contained in $F_{x}$ for some $x \in P$.

Definition 5.1. Let $T$ be a $K P$-algebra. We call a pair $(\Pi, p)$ of a system $\Pi=\left(F_{x}\right)_{x \in P}$ of subsets of a finite set $F$ with $F_{x} \cap F_{y}=\cup_{z \leq x, y} F_{z}$ for every $x, y \in P$ and a surjection $p: T^{\Pi} \rightarrow T$ of $K P$-algebras a presentation of $T$. The homomorphisms induced by $p$ are denoted $p_{Q}: \lim _{Q} T^{\Pi} \rightarrow \lim _{Q} T$ for $Q$ open in $P$ and $p_{x}:\left(T^{\Pi}\right)_{x} \rightarrow T_{x}$ for $x \in P$.

Given a $K P$-algebra $T$ and $x \in P$, the structure-homomorphism from $\lim _{P} T$ to $T_{x}$ is denoted $\pi(T)_{x}: \lim _{P} T \rightarrow T_{x}$.

Definition 5.2. A sectioned KP-algebra $(T, \iota(T))$ consists of a $K P$-algebra $T$ and $K$-linear homomorphisms $\iota(T)_{x}: T_{x} \rightarrow \lim _{P} T$ with $\pi(T)_{x} \circ$ $\iota(T)_{x}=\operatorname{id}_{T_{x}}$ for $x \in P$. 
ExAmple 5.3 (Monomial ideals). Let $I$ be a monomial ideal in the polynomial ring $S=K\left[x_{1}, \ldots, x_{n}\right]$ with irredundant irreducible decomposition $I=\mathfrak{m}^{b^{1}} \cap \cdots \cap \mathfrak{m}^{b^{t}}$. We consider the $K P$-algebra $T=T(I)$ on the poset $P=P(I)$ of Example 2.2. For $D \subseteq C \subseteq[t]$, there is a preferred section $S / \sum_{i \in D} \mathfrak{m}^{b^{i}} \rightarrow S / \sum_{i \in C} \mathfrak{m}^{b^{i}}$ of the projection $T_{D C}$ induced by a $K$-linear homomorphism $S \rightarrow S$ acting either as the identity or as zero on a monomial of $S$. The $K P$-algebra $T$ is sectioned with $\iota(T)_{C}$ such that the composition $\pi(T)_{D} \circ \iota(T)_{C}$ is given by the composition

$$
T_{C}=S / \sum_{i \in C} \mathfrak{m}^{b^{i}} \rightarrow S / \sum_{i \in C \cap D} \mathfrak{m}^{b^{i}} \rightarrow S / \sum_{i \in D} \mathfrak{m}^{b^{i}},
$$

where the first homomorphism is the section described above and the second homomorphism is the natural projection.

The $K P$-algebras of the form $T^{\Pi}$ considered above are sectioned $K P$ algebras with $K$-linear sections $\iota\left(T^{\Pi}\right)_{x}: T_{x}^{\Pi} \rightarrow \lim _{P} T^{\Pi}$ defined by $\iota\left(T^{\Pi}\right)_{x}(f)=\left(p_{y}\left(\iota_{x}(f)\right)\right)_{y \in P}$, where $\iota_{x}: K\left[F_{x}\right] \rightarrow K[F]$ denotes the natural inclusion.

Definition 5.4. We call $(\Pi, p)$ a presentation of the sectioned $K P$-algebra $T$ if it is a presentation of $T$ considered as a $K P$-algebra and the identity $p_{P} \circ \iota\left(T^{\Pi}\right)_{x}=\iota(T)_{x} \circ p_{x}$ holds for every $x \in P$.

The notation of the above definition can be summarized in the following commutative diagram:

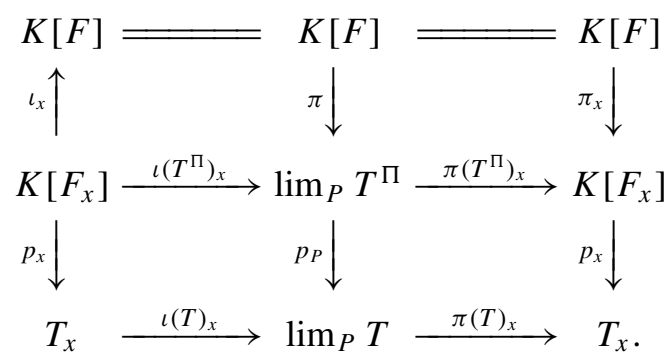

Let us record some facts.

Lemma 5.5. If $(\Pi, p)$ is a presentation of a sectioned KP-algebra $T$, then

(i) $0=\bigcap_{x \in P} \operatorname{Ker}\left(\pi(T)_{x}\right) \subset \lim T$.

(ii) $\operatorname{Ker}\left(p_{P}\right)=\bigcap_{x \in P} \operatorname{Ker}\left(\pi(T)_{x} \circ p_{P}\right)$.

(iii) $\operatorname{Ker}\left(\pi(T)_{x} \circ p_{P} \circ \pi\right)=\operatorname{Ker}\left(p_{x} \circ \pi_{x}\right)=\iota_{x}\left(\operatorname{Ker}\left(p_{x}\right)\right)+\left(F \backslash F_{x}\right)$.

(iv) $\iota_{x}\left(\operatorname{Ker}\left(p_{x}\right)\right)=\operatorname{Ker}\left(p_{x} \circ \pi_{x}\right) \cap \iota_{x}\left(K\left[F_{x}\right]\right)=\operatorname{Ker}\left(\pi(T)_{x} \circ p_{P} \circ \pi\right) \cap$ $\iota_{x}\left(K\left[F_{x}\right]\right)$. 
The following theorem is our main result in this section.

THeOREM 5.6. Let $(\Pi, p)$ be a presentation of a sectioned KP-algebra T . We have that

(i) $\operatorname{Ker}(\pi)=\bigcap_{x \in P} \operatorname{Ker}\left(\pi_{x}\right)=I_{\Delta_{\Pi}}$ is the Stanley-Reisner ideal of $\Delta_{\Pi}$ in $K[F]$.

(ii) $\operatorname{Ker}\left(p_{P} \circ \pi\right)=\sum_{x \in P}\left(\iota_{x}\left(\operatorname{Ker}\left(p_{x}\right)\right)\right)+\bigcap_{x \in P} \operatorname{Ker}\left(\pi_{x}\right)$.

In particular,

$$
\lim _{P} T \cong K[F] / \sum_{x \in P} I_{x}+I_{\Delta_{\Pi}}
$$

where $I_{x}=\left(\operatorname{Ker}\left(p_{x} \circ \pi_{x}\right) \cap \iota_{x}\left(K\left[F_{x}\right]\right)\right)$ for $x \in P$.

Proof. Since we have explained (i) above we only need to prove (ii). Clearly the right hand side is contained in $\operatorname{Ker}\left(p_{P} \circ \pi\right)$. Given $f \in \operatorname{Ker}\left(p_{P} \circ \pi\right)$ we consider the set $P(f)$ consisting of the elements $x \in P$ such that $f$ contains a monomial $m$ with $\operatorname{supp}(m) \subseteq F_{x}$. We prove the opposite inclusion by induction on the cardinality of the set $P(f)$. First note that $P(f)=\emptyset$ implies $f \in \bigcap_{x \in P} \operatorname{Ker}\left(\pi_{x}\right)$. If $P(f) \neq \emptyset$ we choose an element $x \in P(f)$. Since $\iota_{x} \pi_{x}(f) \in \iota_{x}\left(\operatorname{Ker}\left(p_{x}\right)\right)$ the element $f^{\prime}=f-\iota_{x} \pi_{x}(f)$ is an element of $\operatorname{Ker}\left(p_{P} \circ \pi\right)$ with $P\left(f^{\prime}\right)$ a proper subset of $P(f)$. This takes care of the induction step.

In some cases there is a relation between the simplicial complex $\Delta_{\Pi}$ and the $K$-algebra $\lim _{P} T$. In fact, we have:

Corollary 5.7. Let $(\Pi, p)$ be a presentation of a sectioned flasque KPalgebra $T$ consisting of $\mathbf{Z}^{m}$-graded Cohen-Macaulay rings such that $d_{x}<d_{y}$ and $f_{x}<f_{y}$ for $x<y$ in $P$, where $d_{x}=\operatorname{dim}\left(T_{x}\right)$ and $f_{x}=\left|F_{x}\right|$. We have that

$$
\begin{aligned}
\operatorname{depth} \lim _{P} T & =\min \left\{i \in \mathrm{N}: H_{\mathfrak{m}}^{i+\left(f_{x}-d_{x}\right)}\left(K\left[\Delta_{\Pi}\right]\right) \neq 0 \text { for some } x \in P\right\}, \\
\operatorname{dim} \lim _{P} T & =\max \left\{i \in \mathrm{N}: H_{\mathfrak{m}}^{i+\left(f_{x}-d_{x}\right)}\left(K\left[\Delta_{\Pi}\right]\right) \neq 0 \text { for some } x \in P\right\} .
\end{aligned}
$$

In particular, if there exists an $n \in \mathbf{Z}$ such that $\operatorname{dim}\left(K\left[F_{x}\right]\right)=\operatorname{dim}\left(T_{x}\right)+n$ for every $x \in P$ and such that $\operatorname{dim}\left(K\left[\Delta_{\Pi}\right]\right)=\operatorname{dim}\left(\lim _{P} T\right)+n$, then $\lim _{P} T$ is Cohen-Macaulay if and only if $\Delta_{\Pi}$ is Cohen-Macaulay.

Proof. The $K$-algebra $\lim _{P} T^{\Pi}$ is isomorphic to the Stanley-Reisner ring of $\Delta_{\Pi}$. The statements are a direct consequence of [2, Corollary 4.2].

Example 5.8 (Toric face rings). Let $\Sigma$ be a rational pointed fan in $\mathrm{R}^{m}$ and choose affine monoids $M_{C}$ for $C \in \Sigma$ as in Example 2.1. The $K P$ algebra $T$ of Example 2.1 with $T_{C}=K\left[M_{C}\right]$ is a sectioned $K P$-algebra with 
$\iota(T)_{C}: T_{C} \rightarrow \lim _{P} T$ determined by requiring the composition $\pi(T)_{D} \circ \iota(T)_{C}$ to be the composition of the inclusion $K\left[M_{C}\right] \subseteq K\left[M_{E}\right]$ and the face projection $K\left[M_{E}\right] \rightarrow K\left[M_{D}\right]$ if $C$ and $D$ are faces of a common cone $E$ in $\Sigma$, and requiring $\pi(T)_{D} \circ \iota(T)_{C}$ to be zero otherwise. Denoting the Hilbert basis of $M_{C}$ by $F_{C}$, the surjections $p_{C}: K\left[F_{C}\right] \rightarrow K\left[M_{C}\right]$ define a presentation $(\Pi, p)$ of the sectioned $K P$-algebra $T$ with $\Pi=\left(F_{C}\right)_{C \in \Sigma}$. In the particular case where $M_{C}=\mathrm{Z}^{m} \cap C$ for every $C \in \Sigma$, the $K P$-algebra $T$ is denoted $T(\Sigma)$, and $T(\Sigma)_{C}$ is Cohen-Macaulay of $\operatorname{dimension} \operatorname{dim}(C)$ since it is the monoid ring of a normal affine monoid. Note that Corollary 5.7 applies to $T(\Sigma)$.

Finally, we want to compute the initial ideals of the presentation ideal of $\lim _{P} T$ with respect to weight orders $\operatorname{in}_{\omega}$ on $K[F]$ induced by a map $\omega: F \rightarrow \mathrm{R}$.

THeOREM 5.9. Let $(\Pi, p)$ be a presentation of a sectioned KP-algebra T. For a map $\omega: F \rightarrow \mathbf{R}$ we have that

$$
K[F] / \operatorname{in}_{\omega}\left(\operatorname{Ker}\left(p_{P} \circ \pi\right)\right) \cong \lim _{P} K\left[F_{x}\right] / \operatorname{in}_{\omega}\left(\operatorname{Ker}\left(p_{x}\right)\right) .
$$

Proof. The $K P$-algebra $\operatorname{in}_{\omega} T$ with $\operatorname{in}_{\omega} T_{x}=K\left[F_{x}\right] / \operatorname{in}_{\omega}\left(\operatorname{Ker}\left(p_{x}\right)\right)$ is presented by $(\Pi, q)$, where $q_{x}$ is the projection $K\left[F_{x}\right] \rightarrow K\left[F_{x}\right] / \operatorname{in}_{\omega}\left(\operatorname{Ker}\left(p_{x}\right)\right)$. Hence by Theorem 5.6

$$
\operatorname{Ker}\left(q_{P} \circ \pi\right)=\left(\sum_{x \in P} \iota_{x}\left(\operatorname{Ker}\left(q_{x}\right)\right)\right)+\bigcap_{x \in P} \operatorname{Ker}\left(\pi_{x}\right) .
$$

Clearly $\iota_{x}\left(\operatorname{Ker}\left(q_{x}\right)\right)=\iota_{x}\left(\operatorname{in}_{\omega}\left(\operatorname{Ker}\left(p_{x}\right)\right)\right)$ is contained in $\operatorname{in}_{\omega}\left(\operatorname{Ker}\left(p_{P} \circ \pi\right)\right)$, and thus the identity on $K[F]$ induces a homomorphism $K[F] / \operatorname{Ker}\left(q_{P} \circ\right.$ $\pi) \rightarrow K[F] / \operatorname{in}_{\omega}\left(\operatorname{Ker}\left(p_{P} \circ \pi\right)\right)$. On the other hand, $\pi_{x}\left(\operatorname{in}_{\omega}\left(\operatorname{Ker}\left(p_{P} \circ \pi\right)\right)\right) \subseteq$ $\operatorname{in}_{\omega}\left(\operatorname{Ker}\left(p_{x}\right)\right)$, and thus the projection $K[F] \rightarrow K\left[F_{x}\right] / \operatorname{in}_{\omega}\left(\operatorname{Ker}\left(p_{x}\right)\right)$ induces a homomorphism

$$
K[F] / \operatorname{in}_{\omega}\left(\operatorname{Ker}\left(p_{P} \circ \pi\right)\right) \rightarrow K\left[F_{x}\right] / \operatorname{in}_{\omega}\left(\operatorname{Ker}\left(p_{x}\right)\right) .
$$

These homomorphisms in turn assemble to a homomorphism $K[F] / \operatorname{in}_{\omega}\left(\operatorname{Ker}\left(p_{P} \circ \pi\right)\right) \rightarrow \lim _{P} K\left[F_{x}\right] / \operatorname{in}_{\omega}\left(\operatorname{Ker}\left(p_{x}\right)\right) \cong K[F] / \operatorname{Ker}\left(q_{P} \circ \pi\right)$.

We leave it as an exercise to the reader that this is an inverse isomorphisms to $K[F] / \operatorname{Ker}\left(q_{P} \circ \pi\right) \rightarrow K[F] / \operatorname{in}_{\omega}\left(\operatorname{Ker}\left(p_{P} \circ \pi\right)\right)$.

\section{REFERENCES}

1. Alexandrov, P. S.,Combinatorial Topology. Vol. 1, 2 and 3, Transl. from the Russian. Reprint of the 1956, 1957 and 1960 translations. Mineola, Dover Publications (1998). 
2. Brun, M., Bruns, W., and Römer, T., Cohomology of partially ordered sets and local cohomology of section rings, Adv. Math. 208 (2007), 210-235.

3. Brun, M., and Römer, T., Subdivisions of toric complexes, J. Algebraic Combin. 21 (2005), 423-448.

4. Bruns, W., and Gubeladze, J., Polyhedral algebras, arrangements of toric varieties, and their groups, Adv. Stud. Pure Math. 33 (2002), 1-51.

5. Bruns, W., and Herzog, J., Cohen-Macaulay Rings. Rev. ed., Cambridge Stud. Adv. Math. 39 (1993).

6. Caijun, Z., Cohen-Macaulay Section Rings, Trans. Amer. Math. Soc. 349, No. 11 (1997), 4659-4667.

7. De Concini, C., Eisenbud, D., and Procesi, C., Hodge Algebras, Astérisque 91 (1982).

8. Duval, A. M., Free resolutions of simplicial posets, J. Algebra 188, No. 1 (1997), 363-399.

9. Hibi, T., Distributive lattices, affine semigroups rings and algebras with straightening laws, Commutative algebra and combinatorics, US-Jap. joint Semin., Kyoto/Jap. 1985, Adv. Stud. Pure Math. 11 (1987), 93-109.

10. Hibi, T., Quotient algebras of Stanley-Reisner rings and local cohomology, J. Algebra 140, No. 2 (1991), 336-343.

11. Miller, E., and Sturmfels, B., Combinatorial Commutative Algebra, Grad. Texts in Math. 227 (2005).

12. Masuda, M., h-vectors of Gorenstein* simplicial posets, Adv. Math. 194 (2005), 332-344.

13. Munkres, J. R., Topological results in combinatorics, Michigan Math. J. 31 (1984), 113-128.

14. Miyazaki, M., An example of an ASL on a distributive lattice whose straightening relations do not give a presentation, Bull. Kyoto Univ. Ed. Ser. B 83 (1993), 81-84.

15. Stanley, R. P., Balanced Cohen-Macaulay complexes, Trans. Amer. Math. Soc. 249 (1979), 139-157.

16. Stanley, R., Generalized h-vectors, intersection cohomology of toric varieties, and related results, Commutative algebra and combinatorics, Adv. Stud. Pure Math. 11 (1987), 187213.

17. Stanley, R. P., f-vectors and h-vectors of simplicial posets, J. Pure Appl. Algebra 71, No. 2/3 (1991), 319-331.

18. Stanley, R. P., Combinatorics and Commutative Algebra. Second edition, Prog. Math. 41 (1996).

19. Stanley, R. P., Enumerative combinatorics. Vol. 1, Paperback ed. Cambridge Stud. Adv. Math. 49 (1999).

20. Takayama, Y., Combinatorial characterizations of generalized Cohen-Macaulay monomial ideals, Bull. Math. Soc. Sci. Math. Roumanie (N.S.) 48 (2005), 327-344.

21. Trung, N. V., On the presentation of Hodge algebras and the existence of Hodge algebra structures, Comm. Algebra 19, No. 4 (1991), 1183-1195.

22. Yuzvinsky, S., Cohen-Macaulay rings of sections, Adv. Math. 63 (1987), 172-195.

23. Yuzvinsky, S., Flasque sheaves on posets and Cohen-Macaulay unions of regular varieties, Adv. Math. 73 (1989), 24-42.

DEPT. OF MATHEMATICS

UNIVERSITY OF BERGEN

JOHS. BRUNSGT. 12

N-5008 BERGEN

NORWAY

E-mail: morten.brun@math.uib.no
FB MATHEMATIK/INFORMATIK

UNIVERSITÄT OSNABRÜCK

49069 OSNABRÜCK

GERMANY

E-mail: troemer@mathematik.uni-osnabrueck.de 